\title{
Who calls 999 and why? A survey of the emergency workload of the London Ambulance Service
}

\author{
Christina R Victor, Janet L Peacock, Claire Chazot, Sue Walsh, Denise Holmes
}

\begin{abstract}
Background-In 1996-97 there were 623000 emergency (999) calls made to the London Ambulance Service (LAS) and this represents a $30 \%$ increase over the previous five years. The reasons for this increase, which is also observed nationally, remain unknown. It has been suggested that some callers may be using the 999 service "inappropriately" but no data are available from the ambulance service. Objective-To describe the workload of the emergency ambulance service in London with specific reference to the nature and characteristics of 999 calls, to determine who dials 999 and why, and to establish the number and types of calls that could most appropriately be dealt with by other agencies.
\end{abstract}

Design-A one week census of all emergency calls responded to by the LAS. Setting-Sixty eight LAS stations. Methods-Collation of all routine LAS incident forms (LA4) including the classification of the crews' free text description of the incident. This was supplemented by a detailed workload questionnaire for $25 \%$ of calls.

Results-There were 10921 calls responded to from 29 April to 5 May 1996. The census showed that the daily number of calls was highest on Saturday and lowest on Wednesday with about half being made during normal general practitioner (GP) working hours. Half of all calls were for women and one third were for people aged $\geqslant 65$. Accidents were the commonest type of incident $(24 \%)$. The remainder comprised various medical conditions such as respiratory, cardiac, and obstetric problems. In $1.5 \%$ of calls there was no illness, injury, or assistance required and $5 \%$ were for "general assistance" and mostly concerned people aged $\geqslant 65$. The workload survey indicated that two thirds of incidents occurred at home and $70 \%$ of callers had not tried to contact a GP before dialling 999. In the professional opinion of the responding crew, $60 \%$ of calls required a 999 response, with the remainder thought more appropriately dealt with by other services such as primary care, psychiatric services, and social services.

Conclusions-This study suggests that while the majority of 999 calls were "appropriate", part of the 999 workload could be dealt with by other services.
More research is required to clarify why people contact the 999 service for nonemergency incidents and also to establish the views of GPs and other agencies as to the role and function of the LAS. (F Accid Emerg Med 1999;16:174-178)

Keywords: emergency 999 calls; ambulance service; priority despatch

The emergency (999) ambulance service is an important element of the health care system. In 1996-97 there were 2993800 emergency ambulance calls in England. ${ }^{1}$ Within London there were approximately 623000 emergency calls to the London Ambulance Service (LAS), which represents $21 \%$ of calls nationally. The LAS has, like other emergency and health care services, experienced a large increase in workload over the past five years. Emergency 999 calls in England increased by $25 \%$ between 1992-93 and 1996-97 compared with $31 \%$ in London. ${ }^{1}$ The reasons for this increase remain unclear but may reflect increased need for the 999 service from older people, ${ }^{2}$ the decreased availability of other services, or "inappropriate" use of the 999 service (or indeed some combination of these reasons).

Routine LAS data concentrate upon detailing response times (as these are the "quality" measures used in evaluating the service) but these provide little insight into the role and workload of the service. There has been little previous British research describing the role and function of the ambulance service, ${ }^{34}$ although some investigators have attempted to determine the extent of inappropriate use..$^{5-8}$ These studies are of limited use in evaluating the workload of the LAS because they are based upon surveys of patients who arrive at accident and emergency departments and hence exclude those not conveyed to hospital (a group among which inappropriate use may be high). More recently the epidemiology of 999 calls has received some attention within studies looking at the priority dispatch systems, but these studies were either based in nonmetropolitan areas ${ }^{9}$ or confined to paediatric cases. ${ }^{10}$ The applicability of these studies to London is clearly limited.

Studies into the use (and abuse) of ambulance services from other countries are of limited applicability to the UK situation because of the variations between countries in the organisation of health care. A particular problem arises because of international 
differences in whether or not the users pay for the ambulance service.

In 1995 the Wells inquiry into the LAS concluded that there was considerable scope for scientifically sound research into the role, function, and use of the ambulance service in London. ${ }^{11}$ This paper presents the results of an innovative study undertaken by the LAS and St George's Hospital Medical School designed to investigate the current workload of the 999 ambulance service in the capital with specific reference to the nature and characteristics of calls and to determine who calls 999 and why. These data provide a baseline against which to monitor changes in the workload of the LAS resulting from both demographic change and the changing patterns of health care delivery in the capital. Additionally it also provides a comprehensive analysis of the workload which may inform the debate concerning the prioritisation of ambulance response.

\section{Method}

All 999 calls responded to by the LAS during the seven days 29 April to 5 May 1996 by the 68 LAS stations were studied. This excluded hoax calls and calls cancelled by the caller. The study week was not characterised by specific events (extreme weather, major incidents, or large crowd events) that might skew the results.

Two sources of data were used: the routine LAS form (subsequently termed the LA4) and a specially designed one page questionnaire (subsequently referred to as "the workload survey"). The attending ambulance crew completed both forms at the conclusion of each incident.

The LA4 provided information on the age and sex of callers, the date, time, and location of the incident and the hospital to which patients were conveyed. It also includes a free text description of the nature of the incident. This information was not routinely processed at the time and was categorised by one of us (CC).

The workload survey also included standard demographic information (for example age, sex of patient), incident data (time, place of incident and nature of incident, for example road traffic accident, obstetric, psychiatric, etc), who dialled 999 (for example patient, general practitioner (GP), police), why they dialled 999, and attempts to contact other services to deal with incident (for example GP, social services, psychiatric services). It also asked if the patient was registered with a GP and asked who, in the professional opinion of the attending crew, should have dealt with the call (for example LAS, GP, social services, etc). Crews recorded the severity of the incident on a four point scale (life threatening, requires emergency medical treatment, requires treatment, no illness/injury). These categories approximate to the three category classification of 999 calls (immediately life threatening, serious, not life threatening or serious) proposed by the Department of Health. ${ }^{12}$
Table 1 Characteristics of 999 calls 29 April to 5 May 1996 LA4 (routine) data and workload survey; results are number (\%) of calls

\begin{tabular}{|c|c|c|}
\hline Variable & LA4 data & Workload survey \\
\hline \multicolumn{3}{|l|}{ Day of the week } \\
\hline Monday & $1586(14.6)$ & $530(19.1)$ \\
\hline Tuesday & $1536(14.1)$ & $466(16.8)$ \\
\hline Wednesday & $1498(13.7)$ & $451(16.2)$ \\
\hline Thursday & $1549(14.2)$ & $429(15.4)$ \\
\hline Friday & $1571(14.4)$ & $350(12.6)$ \\
\hline Saturday & $1626(14.9)$ & $306(11.0)$ \\
\hline Sunday & $1555(14.2)$ & $244(8.8)$ \\
\hline Total & $10921(100.0)$ & 2776 (99.9) \\
\hline \multicolumn{3}{|l|}{ Time of the day } \\
\hline 0000-0759 & $2260(20.7)$ & $491(17.8)$ \\
\hline $0800-1559$ & $4225(38.7)$ & $1227(44.5)$ \\
\hline $1600-2359$ & $4436(40.6)$ & $1040(37.7)$ \\
\hline Total & $10921(100.0)$ & $2758(100.0)$ \\
\hline \multicolumn{3}{|l|}{ Sex } \\
\hline Male & $5278(50.1)$ & $1265(49.3)$ \\
\hline Female & $5263(49.9)$ & $1301(50.7)$ \\
\hline Total & $10541(100.0)$ & $2566(100.0)$ \\
\hline \multicolumn{3}{|l|}{ Age (years) } \\
\hline $0-14$ & $1110(11.2)$ & 299 (11.5) \\
\hline $15-29$ & $2124(21.4)$ & $466(17.9)$ \\
\hline $30-44$ & $1808(18.2)$ & $526(20.2)$ \\
\hline $45-59$ & $1188(12.0)$ & $309(11.9)$ \\
\hline $60-74$ & $1495(15.1)$ & $357(13.7)$ \\
\hline $75+$ & $2209(22.2)$ & $644(24.8)$ \\
\hline Total & $9934(100.0)$ & $2601(100.0)$ \\
\hline \multicolumn{3}{|l|}{ Patient conveyed } \\
\hline To hospital & $8888(81.4)$ & 2241 (80.7) \\
\hline To home & & $7(0.3)$ \\
\hline Not conveyed & $2032(18.6)$ & $528(19.0)$ \\
\hline Total & $10920(100.0)$ & $2776(100.0)$ \\
\hline
\end{tabular}

\section{Results}

CENSUS OF ALL CALLS

During the study week 10921,999 calls were responded to. Data from LA4 forms were available for all these calls.

\section{Workload of the LAS}

Calls to the LAS were highest on Saturday and lowest on Wednesday (table 1). Calls were concentrated between 8 am to midnight with almost $40 \%$ made between 8 am and 4 pm when, in theory at least, other services are available.

Who uses the 999 ambulance service?

Approximately half of calls were for women (table 1). The majority of calls were for adults with almost a quarter being for people aged $\geqslant 75$ years; $11 \%$ of calls were for children (aged 0-14).

What types of incidents does the LAS see? The types of incidents included diagnostic categories (for example diabetes and epilepsy), symptoms (for example unconscious), and events (for example falls and accidents). The largest percentage of incidents were described as "accidents", while respiratory and cardiac events were major elements of the workload.

\section{WORKLOAD SURVEY}

The workload survey was intended to cover all incidents recorded on the LA4 form and was to be filled out at the completion of the incident. Although not made compulsory by the LAS management a good response was expected because the form had been designed and piloted in conjunction with LAS staff. Survey forms were completed for $2776(25 \%)$ calls. Comparison of the workload survey with the census data indicated that the sample was 
Table 2 Characteristics of incidents; results are number (\%) of calls

\begin{tabular}{lcc}
\hline Variable & LA4 data & Workload survey \\
\hline Nature of call & $2525(23.6)$ & $661(23.8)$ \\
Accident & $753(7.0)$ & $198(7.1)$ \\
Respiratory & $734(6.9)$ & $237(8.5)$ \\
Cardiac & $564(5.3)$ & $122(4.4)$ \\
Obstetric/gynaecological & $546(5.1)$ & $145(5.2)$ \\
Fallen/general assistance & $548(5.1)$ & $132(4.8)$ \\
Assault & $529(5.0)$ & $92(3.3)$ \\
Unconscious/dead & $499(4.7)$ & $249(9.0)$ \\
Gastrointestinal & $337(3.2)$ & $197(7.1)$ \\
Self inflicted injury & $310(2.9)$ & $135(4.9)$ \\
Neurological & $281(2.6)$ & $87(3.1)$ \\
Epilepsy & $241(2.3)$ & $60(2.2)$ \\
Mental illness & $243(2.3)$ & Not recorded \\
Drink related & & \\
Transfer/assisted another & $170(1.6)$ & Not recorded \\
$\quad$ call & $160(1.5)$ & Not recorded \\
No injury/illness & $136(1.3)$ & $44(1.6)$ \\
Diabetic & $2119(19.8)$ & $344(12.4)$ \\
Other & & \\
Medical condition & 0 & $73(2.6)$ \\
not known & $10695(100.0)$ & $2776(100.0)$ \\
Total & & \\
Location of incident & Not recorded & $1728(66.7)$ \\
Home & & $861(33.3)$ \\
Elsewhere & & $2589(100.0)$ \\
Total & & \\
Notal & &
\end{tabular}

Note: totals vary due to missing data.

Table 3 Characteristics of callers and reasons for dialling 999 ( $n=2776)$; results are number (\%)

\begin{tabular}{lc}
\hline Factor & Workload survey \\
\hline Who dialled 999? & $1197(43)$ \\
Friend/relative & $311(11)$ \\
Patient & $273(10)$ \\
Bystander & $230(8)$ \\
GP & $150(5)$ \\
Police/fire & $141(5)$ \\
Public staff & $101(4)$ \\
Social services & $75(3)$ \\
Warden & $72(3)$ \\
Medical staff & $57(2)$ \\
Careline or similar & $12(0)$ \\
Other & $157(6)$ \\
Not known & \\
\hline
\end{tabular}

Note: totals vary due to missing data; percentages do not always add to $100 \%$ due to rounding.

broadly representative of all calls. Weekend and night/evening calls were slightly underrepresented in the workload survey (see tables 1 and 2) as were inappropriate calls. However for the majority of incidents, especially accidents that form a major part of the LAS workload, there was close agreement between the two sets of data. Hence we considered that the workload survey was sufficiently representative of all calls to provide unique additional information concerning the workload of the LAS.

Who dials 999 and why?

Friends or relatives made almost half of calls (table 3). The patient, bystanders, or GPs made the majority of the remaining calls. The vast majority had called an ambulance because "they thought it was necessary".

For the majority of calls the 999 service was the first (and only) one contacted and callers used this service for three main reasons: the perceived severity of the incident, the appropriateness of the incident for a 999 response, or because they did not know what else to do. In a fifth of cases the caller reported that they had contacted the GP and most of these (three quarters) that they were advised by the GP to dial 999 or the GP called the LAS (table 3). Only a small minority of callers $(5 \%)$ subsequently called 999 because of the time they thought the GP would take to arrive or because the GP was reported as not being able (or willing) to visit the patient.

\section{Crews'perceptions of the severity of incidents} attended

Crews rated $8 \%$ of calls immediately life threatening (category 1 ) and $34 \%$ as requiring emergency treatment (category 2) (table 4). A further $46 \%$ of calls were rated as requiring medical treatment but not necessarily a 999 response (table 4). For six incident groupscardiac, respiratory, neurological, unconscious, epilepsy, and diabetes-the majority of incidents were rated as severe (categories 1 or 2) In contrast only a minority of falls, assaults or psychiatric incidents were rated as severe (table 4).

\section{Crews'perceptions of the appropriate agency to} deal with incidents

Crews were asked to determine who, in their professional opinion, was the most appropriate agency to have responded to the incident. Sixty per cent of calls were rated by crews as requiring a LAS 999 response (table 4) and $8 \%$ were seen as "other" types of LAS work. GPs were identified as being the appropriate agency to deal with $13 \%$ of calls and $2 \%$ each for social services, the psychiatric services, and the

Table 4 Assessment of severity and appropriateness of 999 calls by ambulance crews $(n=2776)$; results are number (\%)

\begin{tabular}{|c|c|c|}
\hline \multicolumn{2}{|l|}{ Factor } & Workload survey \\
\hline \multicolumn{3}{|l|}{ Severity of incident } \\
\hline \multirow{2}{*}{\multicolumn{3}{|c|}{ Immediately life threatening }} \\
\hline & & Requires emergency medical treatment via \\
\hline 999 & & $865(34)$ \\
\hline Requires medical tre & & $1174(46)$ \\
\hline No illness or injury & & $307(12)$ \\
\hline \multicolumn{3}{|c|}{ Who should have dealt with incident? } \\
\hline LAS 999 & & $1627(60)$ \\
\hline GP & & $346(13)$ \\
\hline Hospital, but by owr & sport & $252(9)$ \\
\hline LAS as an urgent ca & & 199 (7) \\
\hline Social services & & $60(2)$ \\
\hline Psychiatric services & & $57(2)$ \\
\hline Police & & $53(2)$ \\
\hline District nurse & & $22(1)$ \\
\hline LAS non-urgent cal & & $20(1)$ \\
\hline Other & & $69(3)$ \\
\hline \multicolumn{3}{|c|}{ Severity and appropriateness by nature of incident } \\
\hline Nature of incident & $\%$ Severity $1,2^{*}$ & $\%$ Appropriatet \\
\hline Accidental injury & 48 & 75 \\
\hline Generally unwell & 15 & 29 \\
\hline Gastrointestinal & 29 & 37 \\
\hline Cardiac & 83 & 87 \\
\hline Respiratory & 64 & 76 \\
\hline Self harm & 31 & 51 \\
\hline Neurological & 63 & 72 \\
\hline Assault & 29 & 59 \\
\hline \multicolumn{3}{|l|}{ Obstetric/ } \\
\hline gynaecological & 33 & 50 \\
\hline Unconscious & 69 & 86 \\
\hline Fallen & 13 & 55 \\
\hline Epilepsy & 62 & 88 \\
\hline \multicolumn{3}{|l|}{ Other medical } \\
\hline condition & 16 & 26 \\
\hline Psychiatric & 5 & 7 \\
\hline General assistance & 0 & 19 \\
\hline Diabetic & 59 & 75 \\
\hline
\end{tabular}

Totals vary due to missing data; percentages do not always add to $100 \%$ due to rounding.

^Immediately life threatening or requires emergency medical treatment via 999

† Should have been dealt with by LAS 999 . 
police. There were a greater percentage of appropriate calls (64\%) during "GP hours" than out of hours $(56 \%)$.

The crews' perception of who should have dealt with the call is related to both severity and the nature of the incident. Almost all calls in severity categories 1 and 2 were rated as being LAS 999 calls compared with one third of category 4 calls.

The main types of incidents seen as being appropriate 999 calls were cardiac, respiratory, unconscious, neurological, epilepsy, and diabetic. Although some types of incidents were less likely to be rated by crews as severe, they were still seen as being most appropriately dealt with by the 999 service. Examples of this are accidents, falls, and obstetric incidents. Conversely some incident types, most notably psychiatric calls, were rated by the majority of crews as neither severe in nature nor requiring a 999 response.

\section{Discussion}

The role, function, and workload of the 999 ambulance service in Britain have not been studied extensively. The workload of the emergency ambulance service nationally, and in London in particular, has been increasing. The reasons for this are poorly understood but it is often interpreted as reflecting increased (and possibly inappropriate) demand. However such increases may reflect increased need, resulting from demographic change, or changes in the availability of other health and social care services. Our study utilised two sources of data to evaluate the emergency workload of the LAS for one week in spring 1996. Routine data sources (the computerised LA4 forms) provided data on the temporal variations in the workload. These illustrated that demand on the service is highest on Saturday and approximately half of all calls are made when callers have access to other types of services such as GPs or social care agencies.

Limited comparisons may be drawn between our survey and previously published research. How do the characteristics of people using the 999 service in London compare with previous populations studied? The sex distribution in our study is comparable with previous research but the age profile is distinctive. Morris and Cross reported that $54 \%$ of their sample of 1000 consecutive patients brought to the accident and emergency department by ambulance were male compared with $49 \%$ of our sample. ${ }^{5}$

The percentage of users aged $0-4$ was $6 \%$ in our survey compared with approximately $5 \%$ reported previously. ${ }^{56}$ The distinguishing feature of our population was the high percentage of older people ( $34 \%$ aged $\geqslant 65$ years) compared with $17 \%$ in previous studies. ${ }^{56}$ This does not reflect a higher percentage of older people in London than elsewhere (approximately $15 \%$ of Londoners are aged $\geqslant 65$ and $1.5 \%$ are aged $\geqslant 85$ compared with $16 \%$ and $1.7 \%$ for the nation as a whole). ${ }^{9}$ However a greater percentage of people aged $\geqslant 65$ live alone in London (38\% overall and $42 \%$ in inner London) compared with the general population of England and Wales $(33 \%))^{13}$
Comparing who initiated 999 calls between studies is problematic because of the differing categories used. However the percentage of calls generated by bystanders appears remarkably constant across studies. Morris and Cross ${ }^{5}$ reported that bystanders made $11 \%$ of calls compared with $12 \%$ described by Gardener and $10 \%$ in our study. Our data show that $8 \%$ of calls were made by GPs and $11 \%$ by patients. However virtually no calls in either previous study were reported as being initiated by either patients or GPs. This may reflect either the different ways the data were collected or a real and important change in the identity of people making a 999 call.

Previous studies have not reported why callers dialled 999. In our workload survey the majority of callers opted to dial 999 directly, without contacting other services and did so because they thought it was necessary because of the nature of the incident. With incidents which took place outside the home, 999 calls were often initiated by bystanders or others who did not know the patient. Clearly in such circumstances it is unlikely that they could call the GP rather than 999. However two thirds of incidents occurred at home and it is unclear as to why the GP was not contacted for less serious incidents. There is clearly considerable scope for further research into the help seeking behaviour of users of the 999 service. There may be the potential for the development of educational programmes which will enable callers to determine in which circumstances a 999 call is appropriate and the circumstances when they should contact the GP.

What types of calls are dealt with via the 999 service and how serious are they? The diversity of the workload of the LAS is clearly illustrated by the extensive number of categories required to summarise them. Accidents were the largest single category of incidents and there is clearly little scope for reducing the priority with which such incidents were attended to. Psychiatric disorders accounted for approximately $2 \%$ of the workload and this is broadly similar to previous studies.

While comparatively few calls, $1.5 \%$, required no action in that there was no illness or injury reported by the crew, this still represented 160 calls a week or 8320 a year. Similarly $5 \%$ of incidents (approximately 500 calls a week or 26000 a year) were termed "general assistance". These calls were predominantly from older people who required help after falling (or some other form of general help). These calls represent a clear need for help from older people, perhaps because of the increased prevalence of living alone among older people in the capital or because of the lack of availability of social care agencies. However it is less certain that such calls require a 999 emergency ambulance service response.

Within each element of the health service there have been attempts to enumerate the inappropriate use of facilities. Such studies however sophisticated the methodology, usually rely upon the subjective judgment of the health care professionals involved. Studies of 
ambulance services have attempted to address this issue by looking at either the seriousness of the condition or classifying the appropriateness of calls. In our study we used both approaches. Crews were asked to classify the severity of incidents; $8 \%$ of calls were defined as life threatening compared with $12 \%$ reported by Gardener. ${ }^{6}$ Morris and Cross classified 54\% of their calls as minor, which compares with $58 \%$ of our calls defined as either requires no treatment or requires non-urgent treatment.

Gardener asked patients in his survey to classify the severity of their injury into four categories: life threatening, worried by blood loss/ loss of limb, severe pain, and other. ${ }^{6} \mathrm{He}$ reported that $12 \%$ classified as life threatening compared with $8 \%$ in our survey. Morris and Cross reported that $23 \%$ of their calls were classified by casualty doctors as major, $24 \%$ as intermediate, and the majority $(54 \%)$. as minor. ${ }^{5}$ This later category may approximate to the categories 3 and 4 in our survey, which accounted for $58 \%$ of calls.

In our survey $60 \%$ of calls were defined by crews as appropriate 999 calls. This compares with $48 \%$ justified/possibly warranted, ${ }^{5} 62 \%$ justified, ${ }^{6} \quad 51 \%$ appropriate, ${ }^{7}$ and $56 \%$ appropriate. ${ }^{8}$ Despite the variety of methodologies across studies there is a remarkable similarity in the results with approximately $50 \%$ to $60 \%$ of calls being categorised as appropriate 999 work.

Attempting to determine what, in the opinion of crews, is an appropriate 999 call is clearly problematic. Our data illustrate that appropriateness is not a simple function of severity. While most incidents rated as severe were seen as appropriate 999 calls it does not hold that all non-severe incidents were inappropriate. For example most types of incidents that were rated as serious, such as cardiac calls, were also classed as appropriate for the 999 service to deal with. However some classes of incident which were less likely to be rated as severe, such as falls and accidents, were still seen as being most appropriately dealt with by the 999 service, perhaps because of the potential severity such incidents may pose. Furthermore $19 \%$ of general assistance calls were seen as appropriate even though none of these were rated as severe. Only for psychiatric incidents was there a consistently low severity rating and appropriateness classifications. Such calls were clearly not seen as being the province of the LAS.

Overall crews felt that a 999 response was not required for $40 \%$ of calls. Crews thought that $13 \%$ of calls should have been dealt with by the GP and $19 \%$ by a range of other services such as social services, psychiatric services, district nurses, and the police. However we have not explored the GPs and other agencies' perceptions of this. Neither have we been able to examine how callers perceive the role and function of the LAS. There is a clear need for further research into the relationships between the ambulance service, primary care, and social services departments and into how callers arrive at the decision to dial 999.

Until recently all 999 calls have been responded to in the same way. However the Department of Health has recommended that 999 responses should be prioritised according to the seriousness of the patient's clinical need. Such systems have been studied in the United States and limited research has been undertaken in the UK. The Department of Health estimate that $25 \%$ of calls are in category $\mathrm{A}$, $60 \%$ in category $B$, and $15 \%$ in category $C$; the approximate percentages in these groups for our study were $42 \%, 46 \%$, and $12 \%$ respectively. This difference may result from the Department of Health underestimating the rate of emergencies. Alternatively it may suggest that "true" emergencies form a greater element of the LAS workload than for other areas, perhaps because of the influx of commuters and tourists and the location of major transport termini within the LAS area, and that the increase in use made of the LAS may reflect a "real need" for emergency medical services within the capital.

We wish to acknowledge all the ambulance crews who participated in the survey and the staff at LAS headquarters who helped in the development and implementation of the survey and provided the routine LAS data.

Conflict of interest: none.

Funding: small grant from SE Thames R\&D committee.

1 Department of Health. Statistical bulletin: ambulance services England 1996-97. London: Department of Health, 1997: Englan.

2 Wass A, Zoltien N. Changing patterns in accident and emergency attenders. $\mathcal{F}$ Accid Emerg Med 1996;13:269-71. 3 Boughey WNF. Accidents, emergencies and ambulances. A survey in Portsmouth. BMf 1969;i:369-72.

4 Pugh P. What is under a blue flashing light? Community Medicine 1985;7:198-201.

5 Morris DL, Cross AB. Is the emergency ambulance service abused. BMF 1980;iii:121-3.

6 Gardener GJ. The use and abuse of the emergency ambulance service: some of the factors affecting the decision to call an emergency ambulance. Arch Emerg Med 1990;7:81-9.

7 Pennycook AG, Makower RM, Morrison WG. Use of the emergency ambulance service to an inner city accident and emergency department: a comparison of general practitioner and "999" calls. $f R$ Soc Med 1991;84:726-7.

8 McLeay GF, Hamilton D, Bowie P. Emergency ambulance McLeay GF, Hamilton D, Bowie P. Emergency ambulance News 1995;5(9):137-9.

9 Nicholl J, Gilhooley K, Parry G, et al. The safety and reliability of priority despatch systems. Sheffield: Medical Care Research Unit, University of Sheffield, 1996

10 Williams L, Stephenson T. Prioritisation of ambulance response [letter]. $\mathcal{F}$ Accid Emerg Med 1996;13:231.

11 Wells W. Report on the LAS by the review team to the secretary of state for health following the death of Nasima Begum. London: Department of Health, January 1995.

2 Department of Health. Review of ambulance performance standards. London: DoH, NHS Executive, 1996.

13 Warnes AM. The health and care of older people in London. London: Kings Fund, 1997. 